\title{
Sistema de informações para acompanhamento, controle e auditoria em saúde pública*
}

\author{
Giovanna S. F. Cerchiari** \\ Rolf Hermann Erdmann***
}

SumÁrio: 1. Introdução; 2. Base conceitual; 3. Metodologia; 4. O método proposto; 5. Conclusão.

Summary: 1. Introduction; 2. Conceptual base; 3. Methodology; 4. Proposed method; 5. Conclusion.

Palavras-chave: sistema de informações em saúde; indicadores de efetividade em saúde; custos em saúde pública.

KEY WORDS: health information systems; health effectiveness indicators; public health costs.

Este artigo apresenta alternativas para a gestão dos recursos destinados à saúde pública, por meio de um sistema de informações para os programas de diabetes e hipertensão e resulta do interesse em analisar a efetividade dos programas destinados aos portadores de doenças crônico-degenerativas. As questões analisadas foram focadas em fatores que traduzem a efetividade das ações desenvolvidas, isto é, a capacidade de gerar resultados a partir dos investimentos feitos. Isso ocorreu por meio de um modelo proposto para análise do resultado, mediante o estabelecimento de um indicador que consolidou três frentes de resultado, o número de óbitos,

* Artigo recebido em mar. 2006 e aceito em abr. 2007.

** Mestranda em administração. Pesquisadora da Universidade Federal de Santa Catarina (UFSC). Endereço: Praça Júlio Willerding, 181, apt. 62 - CEP 88301-303, Itajaí, SC, Brasil. E-mail: giovanna@litoral.unimedsc.com.br.

*** Doutor em administração. Coordenador do Programa de Pós-Graduação em Administração da Universidade Federal de Santa Catarina (UFSC). Endereço: Universidade Federal de Santa Catarina, Núcleo Interdisciplinar de Estudos em Gestão da Produção e Custos, campus universitário, Trindade, Caixa Postal 476 — CEP 88040-900, Florianópolis, SC, Brasil. E-mail: erdmann@ cse.ufsc.br. 
internações e atendimentos ambulatoriais. O índice estabelecido para análise da efetividade derivou em um indicador de custos. Como percurso metodológico foi aplicado o modelo teórico de planejamento e controle de produção (PCP) elaborado por Erdmann (1998), que considera sete etapas para a consolidação de informações fragmentadas em um sistema.

Information system for tracking, control and audit in public health

This article presents alternatives to the management of resources destined for public health through an information system for diabetes and hypertension programs. It intends to analyze the effectiveness of the programs focused on chronic and degenerative disease porters. The questions analyzed were centered on factors that translated the developed actions effectiveness, that is, the capacity to get results through investments you have made. We used a model to investigate the results, intervening in the establishment of an indicator that consolidated three fronts of results, the number of deaths, hospitalizations and ambulatory appointments. The established index for effectiveness analysis resulted in a cost indicator. As a methodology we used a theoretical model of Production Control and Plan (PCP), elaborated by Erdmann (1998), which considered seven stages for a consolidation of a fragmented information system.

\section{Introdução}

Este artigo resultou da preocupação com a efetividade do investimento público em saúde. Trata-se de uma abordagem de construção de um instrumento de monitoramento de ações desenvolvidas e dos recursos investidos em programas de acompanhamento de doenças crônico-degenerativas por meio de um sistema de informações gerenciais. Consiste em desenvolver um estudo baseado em análise de custo e efetividade das intervenções assistenciais em doenças crônico-degenerativas. A referência utilizada foi a cidade de Itajaí-SC.

Os objetivos específicos consistem em estabelecer parâmetros para serem utilizados como indicadores de efetividade dos programas desenvolvidos para os portadores de doenças crônico-degenerativas e conceber um método para análise da efetividade desses programas.

Programas que visam a prevenção, controle e tratamento de algumas doenças estão em andamento em diversas regiões, de acordo com as características populacionais. A questão a discutir é se os programas existentes estão apresentando resultados positivos com as ações desenvolvidas. O programa de diabetes e hipertensão é direcionado para um grupo portador de doenças crônico-degenerativas. Buscou-se avaliar a qualidade dessas ações por meio do resultado provocado pelo programa. Os programas são financiados pelo 
Sistema Único de Saúde. A proposta objetivou acompanhar os resultado e os investimentos realizados.

Neste artigo, apresentamos um método que permite identificar resultados, por meio de indicador de desempenho em programas específicos. A partir desse indicador poderá ser avaliado como os recursos estão sendo aplicados e qual a sua efetividade. Isso decorre da qualidade dos atendimentos prestados, da eficiência da assistência preventiva (diminuição da incidência de internações hospitalares e óbitos) e necessidades não supridas da comunidade.

A evolução da incidência das doenças está relacionada aos serviços de saúde disponíveis para a população. Algumas patologias tornaram-se crônicas, a morbidade e a mortalidade decorrentes delas agravaram-se, e com isso as conseqüências da necessidade de programas de saúde que revertam a situação, em busca do equilíbrio e manutenção da saúde, e acompanhamento dos doentes.

Programas destinados a portadores de doenças crônico-degenerativas são desenvolvidos em diversas regiões do país. Neste artigo focamos o município de Itajaí-SC, por gozar de autonomia (gestão plena) dos recursos vindos da esfera federal e, secundariamente, por ter proporcionado acesso.

A questão a se discutir é a efetividade dos programas existentes, direcionados aos portadores de doenças crônico-degenerativas.

Buscamos desenvolver um instrumento que permitisse estabelecer relações entre as ações e os esforços em programas de diabetes e hipertensão arterial, e os respectivos resultados. Relacionamos as variáveis de mortalidade por causa específica, atendimentos ambulatoriais, internações hospitalares e verbas repassadas para o desenvolvimento das ações.

\section{Base conceitual}

Nesta seção discutiremos as relações entre saúde e doença, a epidemiologia relacionada às doenças crônico-degenerativas, as informações em saúde, importância do planejamento, controle e avaliação, custos em saúde, indicadores e o Sistema Único de Saúde (SUS).

A saúde deve ser analisada desde os conceitos até as questões que envolvem as características apresentadas pela população. Trata-se de verificar como a população está enquadrada em termos de saúde e como a gestão pública desenvolve as ações para buscar a saúde dela.

O assunto é complexo e não há precisão e unanimidade sobre a matéria. Buss (1995) comenta sobre as dificuldades em discutir as condições de saúde 
no Brasil, as deficiências dos indicadores comumente utilizados para medi-la e a precariedade das informações disponíveis sobre morbidade e mortalidade. A pesquisa contempla o contexto de ações desenvolvidas em um programa direcionado para os acometidos por diabetes e hipertensão arterial.

A epidemiologia (estudo dos fatores que determinam o surgimento, freqüência, distribuição, evolução, medidas de controle, erradicação e prevenção das doenças) citada por Dever (1998:48), "pode e deve ser usada para fins de administração dos serviços da saúde. Ela contribui para fazer o diagnóstico de uma comunidade da presença, natureza e distribuição de saúde e doença". Dessa forma é possível monitorar a saúde da população e projetar mudanças de acordo com a necessidade.

Ao estudar as mortes por doenças crônicas, deveriam ser analisadas as causas múltiplas, pois na maioria das vezes há uma associação. Porém não é possível fazer essa análise com os dados nacionais porque são publicadas apenas as causas básicas (Cianciarullo, 1998).

Em vista da situação epidemiológica, dados de mortalidade e morbidade, os programas de "controle de doenças" do Ministério da Saúde são desenvolvidos nos municípios; indicam grupos de doenças nos quais são trabalhados a prevenção, diagnóstico e tratamento.

O Ministério da Saúde, por meio dos municípios, exerce a responsabilidade na atuação em saúde, desde a prevenção, diagnóstico e tratamento das patologias, bem como fornecimento de alguns medicamentos e orientações diversas.

As doenças crônico-degenerativas apresentam alta incidência, e necessitam ser acompanhadas as informações relacionadas ao desenvolvimento de ações para o controle delas, incidência, resultados obtidos com as ações, mortalidade, e demais fatores sociais dos portadores dessas doenças.

Por isso, as informações em saúde são essenciais para o início e continuidade das ações, bem como a divulgação das mesmas. Pelas mais diversas formas ocorre a divulgação dessas informações.

Moraes (1994) comenta sobre a origem das informações consideradas relativas à saúde, que começaram a ser trabalhadas no território nacional, em 1931, pela Diretoria Geral de Informações, Estatística e Divulgações do Ministério da Educação e Saúde, no Rio de Janeiro, mais tarde transformada no Serviço de Estatística da Educação e Saúde. Posteriormente as informações passaram a ser atribuição do Instituto Brasileiro de Geografia e Estatística (IBGE), em meados de 1937. Em 1974, o presidente Ernesto Geisel, juntamente com os ministros Mario Henrique Simonsen (Fazenda), João Paulo dos Reis Velloso (Planejamento) e Luiz Gonzaga do Nascimento e Silva (Previdência 
Social), assinaram a Lei no 6.125 , de 4 de novembro, que autoriza o Poder Executivo a constituir a empresa Dataprev, com a finalidade de análise de sistemas, programação e execução de serviços de tratamento da informação e o processamento de dados por meio da computação eletrônica, bem como a prestação de outros serviços correlatos. Mais tarde viria a assumir a implementação da maior base de dados relacionados à área social.

As informações em saúde são essenciais para a realização do planejamento das ações em saúde. Conforme Tinoco (1984), o planejamento pode ser um processo mediante o qual se lança o desenvolvimento, da forma mais racional e rápida possível.

O mesmo autor destaca que os administradores de sistemas de saúde dispõem atualmente de recursos técnico-administrativos que lhes permitem atuar com mais segurança na solução dos problemas em saúde. Com o processo de planejamento implantado, podem ser utilizadas técnicas que lhes facilitem a tomada de decisão. Alguns fatores estão diretamente relacionados à aplicação dessa técnica, podendo apresentar maior ou menor efetividade. Entre os fatores, podemos destacar:

v a informação estatística disponível deve ser em qualidade e quantidade adequadas;

v conhecimento epidemiológico - apesar de avançado, não se apresenta em extensão suficiente para abranger todas as doenças e agravos à saúde;

v esforço metodológico despendido na operacionalização das técnicas - necessidade de pessoal especializado.

Para estabelecer o diagnóstico de uma situação de saúde, a matéria-prima básica é a informação estatística. Essa matéria-prima imprescindível para o diagnóstico também o é para a elaboração do plano, para a avaliação e o controle dos resultados dos programas desenvolvidos.

Para alcançar tais objetivos, foi atribuída ao departamento de informática do SUS, Datasus, a responsabilidade de coletar, processar e disseminar informações sobre saúde. Assim, podemos destacar que as informações em saúde viabilizam o acesso ao conhecimento da realidade existente com cada grupo de doenças, em cada grupo da população (regiões ou estados). Por meio desse conhecimento, pode-se analisar a situação atual, planejando ações, controlando e avaliando resultados alcançados.

O atual sistema de informações do Ministério da Saúde é o Datasus, e nele é possível coletar informações a respeito de produção ambulatorial, internações hospitalares, recursos financeiros repassados, indicadores de 
saúde de cada região por tipo de doença ou outro fator, relatórios de gestão, entre outros.

Algumas questões relativas a informações em saúde podem ser observadas a partir de informações estatísticas. Estas informações estatísticas auxiliam no planejamento, controle e avaliação das questões relacionadas à situação levantada.

Controlar assegura que o desempenho corresponda aos planos, os resultados são medidos e confrontados com padrões, e quando necessário tomadas ações corretivas (Martins, 2000).

Chiavenatto (1994) relata sobre o controle, que é a função administrativa que consiste em medir e corrigir o desempenho dos subordinados para assegurar que os objetivos da empresa sejam atingidos.

A Organização Mundial de Saúde (OMS) definiu, em 1981, como objetivos da avaliação no processo de desenvolvimento dos sistemas de saúde, a melhoria dos programas e dos serviços e a orientação para a distribuição dos recursos humanos e financeiros entre os serviços atuais e futuros. Deve-se empregar a avaliação de maneira construtiva, é essencial que seja concebida como um instrumento para a adoção de decisões estritamente vinculadas com o processo (Secretaria de Estado de Santa Catarina, 1994).

As ações desenvolvidas pelo Sistema de Avaliação em Saúde devem ter por objetivo a análise da qualidade, produção e custos dos serviços produzidos em todos os níveis de controle da execução das ações em saúde, previstas nos planos e programas do governo e de investimento setorial, além do estudo do desempenho gerencial dos órgãos gestores e dos sistemas locais e regionais de saúde (Secretaria de Estado de Santa Catarina, 1994).

A auditoria é vista atualmente como um instrumento de controle administrativo que se destaca como ponto de convergência de todos os feitos, fatos e informações no sentido de que sejam confiáveis, adequados, totais e seguros (Secretaria de Estado de Santa Catarina, 1994).

A avaliação dos programas de saúde deve incorporar a abordagem da avaliação econômica. A complexidade crescente dos sistemas de saúde e o reconhecimento de que as necessidades de saúde são inesgotáveis exigem que se avalie a relação entre os recursos utilizados e a prestação de serviços ou atividades desenvolvidas. É importante considerar que nem todos os benefícios decorrentes de serviços, programas ou atividades de saúde desenvolvidos podem ser quantificados. Esse é o limite da avaliação econômica (Tanaka, 2001).

A partir das informações estatísticas é possível realizar o planejamento, o controle e a avaliação dos serviços de saúde. O planejamento deve ser realizado de acordo com a necessidade local, recursos disponíveis, objetivos 
propostos, entre outros. A avaliação pode ser considerada uma das partes do planejamento. O controle é o processo de avaliação dos serviços e ações em relação ao que foi planejado; tem como objetivo medir e corrigir o desempenho e assegurar que os planos sejam alcançados.

Em saúde, é indispensável o acompanhamento dos custos decorrentes dos serviços e ações desenvolvidas. Uma dificuldade reconhecida na avaliação econômica diz respeito à necessidade de calcular o custo unitário da atividade ou procedimento. Os custos dizem respeito ao consumo de tudo que é incorporado ao produto (Tanaka, 2001).

Sob o ponto de vista econômico, custo significa aplicações de recursos, sob diferentes formas e expressa em seu valor monetário, para produção ou prestação de serviços. Em qualquer administração, o controle dos custos determina a análise sistemática das variações, tanto em seu aspecto numérico, quanto nos motivos que as tenham determinado. O cálculo dos custos deve ser o mais completo e exato possível, para servir de meio de conhecimento das condições econômicas dinâmicas e de controle de produtividade e eficiência administrativa (Lima, 1979).

A análise dos custos é essencial para o controle dos programas desenvolvidos em saúde. Para tal análise deve-se considerar todos os fatores envolvidos nos processos em questão. Além dos custos, um fator importante é o aproveitamento adequado dos recursos, ou seja, por meio do planejamento, coordenação, direção e controle.

Em procedimentos de avaliação, os indicadores proporcionam visualização de tendências e resultados dos fatores a serem analisados. Com o auxílio de indicadores hospitalares torna-se possível avaliar se os programas e os serviços hospitalares estão influenciando de maneira positiva na saúde e na população, ao mesmo tempo em que também evidenciam a qualidade e a quantidade produzidas, bem como a imagem do institucional e nível de satisfação do cliente.

Segundo Tanaka (2001), o indicador é uma variável, característica ou atributo de estrutura, processo ou resultado que é capaz de sintetizar e/ou representar e/ou dar maior significado ao que se quer avaliar.

Para avaliar o grau de saúde da população estabeleceram-se níveis de saúde que permitem a comparação de coeficientes obtidos em outras áreas e períodos. Para isso, são utilizados indicadores que mensuram o grau de saúde (Moraes, 1994).

Um indicador importante para avaliação das questões em saúde é a mortalidade. A mortalidade pode ser analisada por meio do Sistema de Informações de Mortalidade (SIM), disponibilizada pelo Ministério da Saúde e pela 
Secretaria de Estado de Saúde. Permite o conhecimento dos óbitos no tempo e espaço geográfico, causas, idade e sexo. Os dados sobre mortalidade são os principais subsídios para avaliação do serviço prestado. Independentemente de processamento informatizado, os gestores podem coletar seus dados no cartório de registro civil e selecionar algumas variáveis a serem trabalhadas (Secretaria de Estado de Santa Catarina, 1994).

A ocorrência de internações devido às doenças em questão traduz-se em um importante indicador sobre a efetividade dos programas de diabetes e hipertensão. Para avaliar um serviço de saúde, pode-se utilizar as informações sobre a produção ambulatorial do local ou região a ser estudada.

A análise da efetividade de um programa pode ser simplificada adotando-se o critério de proporções envolvendo as três variáveis: recurso, atividade e objetivos (Tinoco, 1984). Segundo Valla (1993), a luta por um sistema de saúde que atenda a todos de modo eficaz, protegendo e recuperando os que adoecem, de modo igualitário e gratuito, pode ser conduzida também como luta para que esse mesmo sistema funcione para prevenir e evitar a doença.

Pela análise dos indicadores é possível avaliar um serviço ou programa de saúde. Alguns indicadores de maior importância são os de mortalidade, morbidade e custos. Para a análise dos indicadores é necessário o conhecimento de todas as variáveis relacionadas ao indicador, procedência, importância, facilidade e aplicabilidade.

O conhecimento da estrutura e dos fatores operacionais dos serviços de saúde é essencial para a escolha dos indicadores a serem utilizados. O Sistema Único de Saúde é responsável por todos os atendimentos e recursos destinados gratuitamente para a saúde da população.

Em 1988, a Constituição Federal, então promulgada, instituiu o Sistema Único de Saúde (SUS), com direção única em cada esfera de governo. Dois anos após a promulgação da Constituição, a Lei $n^{0} 8.080 / 90$ e a Lei $n^{\circ}$ 8.142/90 foram aprovadas.

O gerenciamento da prestação e controle dos serviços e ações de saúde, para que sejam garantidas a gratuidade, universalidade, eficácia e eficiência, deve ser participativo, democrático e descentralizado (Estado de Santa Catarina - Secretaria de Estado da Saúde, 2000).

Quanto ao gerenciamento participativo, significa permitir o controle e intervenção de todos os cidadãos na forma de como estão sendo prestados os serviços de saúde à comunidade em geral. O gerenciamento democrático deve atender aos interesses dos usuários, quanto às suas reais necessidades de serviços de saúde. O gerenciamento descentralizado deve oferecer condições materiais, físicas, financeiras, de recursos humanos e políticas, para permitir a 
administração e o controle em cada esfera do governo (municípios, estados e União), de forma autônoma e com poder de decisão.

Nos arts. 8o e 9o da Lei Orgânica da Saúde, no princípios na organização e direção do SUS:

$\checkmark$ regionalização e hierarquização — os serviços de saúde estão classificados em níveis de complexidade crescente, e alguns serviços necessitam de toda estrutura e condições especiais para sua execução, outros não;

v resolutividade - a garantia da solução dos problemas tanto do indivíduo quanto da coletividade, conforme seu nível de competência;

v descentralização - entendida como a redistribuição das responsabilidades nos três níveis de governo, implica que quanto mais próximo do problema, melhores condições na resolução deles. Assim, o que for de abrangência municipal deverá ser de responsabilidade do governo municipal, na abrangência do estado, do governo estadual e o de nacional será de responsabilidade federal;

v participação dos cidadãos - garantida pela Constituição Federal e regulada pela Lei no 8.142/90, significa que a população participará do processo de formulação das políticas de saúde e do controle da sua execução, nos níveis municipal, estadual e federal, por meio dos conselhos e conferências de saúde;

v complementaridade do setor privado - o SUS poderá recorrer ao setor privado, desde que comprovada a insuficiência de sua rede. Os arts. 25 e 26 da Lei no 8.080/90 estabelecem as condições em que se dará a contratação desses serviços.

A Lei no 8.080/90 comenta também sobre a gestão do SUS. As normas operacionais básicas (NOBs) são parte do conjunto de leis do SUS, regulamentam os processos de descentralização da gestão dos serviços e das ações de saúde.

Foram formuladas três NOBs, em 1991, 1993 e 1996. Elas têm sido resultado de amplas discussões e negociações entre representantes dos governos federal, estadual e municipal e conselhos de saúde.

Tendo como referência a Norma Operacional Básica no 01/96, municipalização significa dar ao município a responsabilidade de definir e gerir as ações e serviços de saúde e a possibilidade de controle da sociedade sobre a responsabilidade dos gestores e a efetiva participação social na formulação de estratégias e ações locais de saúde. A municipalização possibilita a autonomia de gestão municipal, o uso e a redistribuição mais eficiente dos recursos, me- 
lhor ordenação de serviços públicos, facilitando o controle social e ampliando os direitos sociais.

A partir da NOB/96, os municípios puderam habilitar-se em duas condições: gestão plena de atenção básica ou gestão plena do sistema municipal, de acordo com a capacidade dos municípios em assumirem e cumprirem responsabilidades perante a população, o estado e o Ministério da Saúde.

Na gestão plena do sistema municipal, o município recebe de forma automática os recursos financeiros para prestar ações e serviços da assistência ambulatorial e hospitalar. Envolve o planejamento, controle e avaliação, com gerenciamento de todas as unidades ambulatoriais básicas especializadas e hospitalares, públicas ou privadas, vinculadas ao SUS em seu território.

Referente ao tipo de gestão na esfera estadual, a gestão plena do sistema estadual, na qual Santa Catarina habilitou-se desde 1o de janeiro de 2000, é o tipo de gestão em que o estado recebe, de forma regular e automática, os recursos financeiros para gerenciamento do sistema de saúde no âmbito estadual, cooperando com recursos técnicos e financeiros, junto aos municípios. O ingresso nessa modalidade de gestão requer $80 \%$ dos municípios habilitados, ou $50 \%$, desde que somem $80 \%$ da população estadual.

O SUS é financiado com recursos das três esferas de governo. É atribuição constitucional dos poderes público municipal, estadual e federal destinarem uma parcela de seu orçamento para ações e serviços de saúde. É composto pelo orçamento da seguridade social (contribuições dos empregadores incidentes sobre a folha de salários, faturamento e o lucro; dos trabalhadores e sobre a receita de concursos de prognósticos (loterias oficiais), e orçamento fiscal da União, estados e municípios.

Os recursos para saúde, repassados pelo Tesouro Nacional e seguridade social, geridos pelo Ministério da Saúde, são divididos em duas partes: uma é retida para o custeio e investimentos das ações federais; a outra é repassada às secretarias de Saúde estaduais e municipais, de acordo com critérios previamente definidos em função da população, necessidades de saúde e rede assistencial.

Em setembro de 2000, foi aprovada pelo Congresso Nacional a Emenda Constitucional ํo 29 que vincula, no caso dos estados e municípios, recursos advindos de receitas de impostos para a manutenção e desenvolvimento das ações e serviços de saúde de maneira semelhante ao que já acontece com a educação. O valor desse recurso é de $12 \%$ das receitas de impostos no caso dos estados e Distrito Federal e de $15 \%$ no caso dos municípios, e quem estiver aplicando um percentual inferior ao estipulado terá até o exercício financeiro de 2004 para atingi-lo. 
A Constituição Federal foi alterada em seus arts. 34, 35, 156, 160, 167 e 198, passando a União, os estados e os municípios a terem em seus orçamentos percentuais vinculados à saúde.

O sistema de informações sobre orçamentos públicos em saúde (Siops) será utilizado para subsidiar o acompanhamento. A parcela de custeio da esfera federal para assistência ambulatorial e hospitalar configura um teto financeiro global para cada estado e município. $\mathrm{O}$ teto financeiro do estado contém os tetos dos municípios (em qualquer gestão). É composto por:

- PAB - piso de atenção básica é o montante de recursos destinados ao custeio de procedimentos e ações de assistência básica de responsabilidade tipicamente municipal. O município recebe o recurso independentemente do quantitativo da produção;

- MAC - média e alta complexidade ambulatorial é o montante de recursos que corresponde aos procedimentos ambulatoriais apurados pelo sistema de informações ambulatoriais (SIA/SUS), tais como exames laboratoriais, raios X, medicamentos e insumos excepcionais, órteses e próteses ambulatoriais, ultra-som, terapia renal substitutiva, oncologia e tratamento fora do domicílio de estado. O valor do teto financeiro para a MAC é pactuado e definido de acordo com critérios preestabelecidos na comissão bipartite (CIB);

v AIH - autorização de internação hospitalar consiste no pagamento dos valores apurados por intermédio do sistema de informações do SUS (SIH/ SUS), englobando o conjunto de procedimentos realizados em regime de internação hospitalar, com base na autorização de internação hospitalar (AIH), documento de autorização e faturamento dos serviços. É quantificada de acordo com o número de habitantes do município.

Os recursos do $\mathrm{PAB}$, variáveis e fixos, são creditados em uma mesma conta e deverão estar identificados nos orçamentos municipais como receita de transferências intergovernamentais. Os recursos do MAC e AIH, destinados aos municípios em gestão plena de atenção básica, são transferidos diretamente às unidades prestadoras de serviços, conforme programação definida na comissão intergestores bipartite ou diretamente ao fundo municipal de saúde, quando o serviço oferecido for da rede própria.

Para aplicação dos recursos, o plano municipal de saúde é o instrumento que disciplinará a destinação dos recursos, por ser o documento que explicita as intenções do gestor no período de governo específico e aprovado pelo Conselho Municipal de Saúde. 
As informações sobre os recursos financeiros repassados para o fundo municipal de saúde e os dados estatísticos sobre atendimentos estão disponíveis no site do Ministério da Saúde, pesquisas nos links disponíveis. Algumas informações também podem ser coletadas nas secretarias municipais de Saúde.

\section{Metodologia}

A problemática em questão é pouco conhecida e começa mostrando a estrutura existente na Secretaria Municipal de Saúde de Itajaí-SC, pelo prisma das ações desenvolvidas com os portadores de patologias crônico-degenerativas, analisando os custos e efetividade dos programas, a fim de propor um método para o acompanhamento, controle e auditoria.

A abordagem da pesquisa apresenta enfoque qualitativo e quantitativo. Trata-se de avaliar as relações entre as variáveis. Objetivando o desenvolvimento de um método para análise da efetividade dos programas de acompanhamento de doenças crônico-degenerativas e comparar os custos despendidos com os resultados obtidos, foi proposta a utilização de alguns indicadores para a avaliação quantitativa desses programas, com base em premissas de ordem qualitativa. Com base nos objetivos essa pesquisa é exploratória.

A população analisada foi a Secretaria Municipal de Saúde do Município de Itajaí (SC). Como amostra, podemos indicar os programas desenvolvidos com os portadores de doenças crônico-degenerativas, programas de diabetes e hipertensão.

O modelo para análise da situação dos programas desenvolvidos para os portadores de patologias crônico-degenerativas teve o percurso metodológico baseado no modelo proposto por Erdmann (1998), que parte de um levantamento de informações sobre a realidade atual em vários aspectos (diferenciação), permitindo delinear um sistema (integração), para se avaliar e conceber um recurso auxiliar. Essa proposta orientou-se por uma composição de informações das partes para o todo, do disperso para o consolidado.

As linhas básicas para o modelo proposto por Erdmann (1998) são compostas de sete etapas: diagnóstico da situação; processo decisório na concepção do sistema; definição e descrição dos subsistemas; avaliação e descrição detalhada; concepção ou união dos subsistemas; adequação de um software ao modelo, e orientação para implantação. Destas, foram utilizadas aqui as cinco primeiras etapas. 


\section{0 método proposto}

Foi aplicado o modelo proposto com os dados apresentados pela Secretaria Municipal de Saúde de Itajaí.

\section{Diagnóstico da situação}

Foi realizado o levantamento da situação atual dos programas desenvolvidos para os portadores de diabetes e hipertensão no município de Itajaí-SC, por meio de entrevistas com gestores e coleta de dados estatísticos. Foi aplicado um instrumento de entrevista que permitiu o conhecimento da estrutura administrativa e técnica, ações desenvolvidas e necessidades apontadas pelos entrevistados (gestores, participantes dos programas). Foi possível identificar a necessidade de informações gerenciais sobre os programas desenvolvidos. Os gestores desconhecem indicadores que reflitam a atual situação das ações desenvolvidas e se estão alcançando os objetivos propostos.

Assim, optou-se por identificar os problemas detectados e as sugestões apontadas, a fim de verificar a disponibilidade dos dados e a relevância dos mesmos, para a definição dos subsistemas que atenderão as informações apontadas.

\section{O processo decisório na concepção do sistema}

Deve-se estabelecer os objetivos de acordo com as necessidades e expectativas das pessoas, da situação, das contingências e dos problemas identificados. Após o levantamento da situação atual, os problemas identificados relacionados ao desenvolvimento dos programas de diabetes e hipertensão subsidiaram o desenvolvimento do sistema de informações para os programas de diabetes e hipertensão (SIPDH).

Como o objetivo do SIPDH será atender às necessidades e dúvidas apontadas na identificação da situação atual, as respostas coletadas nas entrevistas e as questões sem respostas até o momento reforçam o desenvolvimento desse sistema de informações.

O instrumento deve possibilitar a análise anual, com acompanhamentos mensais ou semestrais; deve resultar em um indicador quantitativo, ou seja, um número; ter relação com os recursos financeiros investidos; proporcionar visão da evolução; contemplar os dados sobre números de óbitos e internações 
por causa específica, permanência hospitalar, recursos investidos; deve, enfim, permitir visualizar uma relação entre o esforço despendido (dinheiro) e o resultado obtido (ações para controle das doenças).

\section{A definição e descrição dos subsistemas}

A partir da visão geral, buscou-se definir os subsistemas baseados nos objetivos do sistema, dos procedimentos empregados, dos modelos já existentes para analisar os resultados que o sistema deve produzir, as saídas.

Por meio de uma análise retroativa, de quais subsistemas atenderão às saídas anteriormente esperadas, seguiu-se para o envolvimento de todas as atividades componentes do sistema. Buscou-se o conhecimento das necessidades e dificuldades específicas relacionadas às informações e no desenvolvimento dos programas de diabetes e hipertensão, e também identificação de quais resultados o sistema de informação necessitou produzir.

Assim, foram apresentados os indicadores para análise dos programas, buscando o relacionamento de dados estatísticos sobre a mortalidade, período de internação hospitalar, atendimentos ambulatoriais e recursos financeiros.

\section{Avaliação dos subsistemas e sua descrição detalhada}

Conforme Erdmann (1988), deve-se avaliar em conjunto com os envolvidos no processo, a forma e os resultados propostos. Foram apresentadas as fórmulas e realizada a explicação dos cálculos utilizados para a análise dos indicadores. Nessa etapa, a fim de aprofundar o conhecimento sobre as informações e o desenvolvimento dos programas de diabetes e hipertensão, foi detalhado cada indicador citado na etapa anterior, sua aplicação e limitações, objetivando o conhecimento da situação dos programas de diabetes e hipertensão.

Os indicadores definidos para a análise da efetividade dos programas são:

v indicador de mortalidade por causa específica - determina o número de óbitos decorrentes da diabetes e hipertensão. Esses dados devem ser obtidos, considerando as variáveis de um mesmo período e local (município). Os dados sobre mortalidade estão disponíveis no site do Ministério da Saúde (Datasus). Entende-se por óbitos a soma de casos de óbitos devido a diabetes e hipertensão, analisados por meio das estatísticas do grupo CID10, do I 20 a I 25, e I 60 a I 69, e para os casos de diabetes mellitus, os CIDs E 10 a E 14 (Rede Interagencial de Informações para a Saúde - Ripsa, 
2002). Para a identificação dos óbitos por causa específica foi utilizada a codificação apresentada no relatório do Departamento de Controle e Avaliação, lista de óbitos conforme a causa. Os óbitos devido à diabetes mellitus seguiram a codificação 055 , e as causas de óbitos relacionados ou causados pela hipertensão (doenças do aparelho circulatório) foram identificadas pela codificação 067, 068, 069, 070, 071. Esse indicador demonstra o índice de mortalidade ocasionado pela diabetes e pela hipertensão, refletindo o estado de saúde da população em estudo. Se o índice apresenta-se elevado, as estruturas dos programas de acompanhamento e controle dessas patologias necessitam ser revistas, bem como a participação da população e as ações desenvolvidas. Esse indicador reflete a efetividade dos programas. Índices baixos de mortalidade são encontrados onde a população não morre dessas doenças. Com isso, pode-se concluir que as ações desenvolvidas nos programas atingem a população-alvo, não ocorrendo ou reduzindo o risco de óbitos por essas causas;

- indicador de internação por causa específica — os números utilizados para a quantidade de dias de internações foram agrupados conforme a ocorrência de internações pelas causas de diabetes e hipertensão, conforme mencionado anteriormente, com os CIDs associados. Para definição da permanência hospitalar foram selecionados os códigos do programa Datasus referentes aos procedimentos relacionados às doenças diabetes e hipertensão arterial: 773000068, 7730050, 77500024, 77500032, 77500105, 77500113, 77500121, 77500130, 77500164, 81500106, 82300038, 82300046, $82500045,82500053,84500042$. Foram somados os dias de internações de acordo com cada codificação, ou seja, cada código apresenta um período de internação em dias preestabelecidos. A alta incidência de internações devido a diabetes e hipertensão indica que a população está suscetível a essas patologias. Logo, esse indicador permite avaliar a efetividade das ações. A baixa incidência levará a uma interpretação de ações bem desenvolvidas, população bem atendida, consciente etc. Com esse indicador será possível analisar o impacto social decorrente das internações e o grau de complicações dessas patologias;

- indicador sobre os serviços prestados ao diabético e hipertenso em nível ambulatorial - buscou-se analisar, em conjunto com a coordenadora das unidades de saúde, os atendimentos relacionados aos portadores de diabetes e hipertensão. Por não haver registro específico dos atendimentos ambulatoriais realizados pelo grupo de diabéticos e hipertensos, foram identificados os atendimentos e suas respectivas produções que podem ser considerados 
realizados pelo grupo em questão. Quanto maior esse índice, melhor o desempenho em relação às ofertas de serviços de prevenção, diagnóstico e tratamento ambulatorial. A limitação na escolha desse indicador decorre do fato dos registros nas unidades serem realizados para toda a comunidade. Alguns códigos são específicos para os diabéticos e hipertensos, para outros atendimentos é usada a mesma codificação dos outros membros da comunidade.

\section{A concepção do sistema}

Por meio das entradas e saídas já definidas, o sistema foi esquematizado com todas as interligações pertinentes. Com os subsistemas integrados, os resultados que o sistema produzirá poderão ser observados. A concepção do sistema deu-se pela união dos subsistemas desenvolvidos, dentro das possibilidades apresentadas de informações disponíveis.

Como objetivo do modelo pode-se destacar: integração e disponibilidade de informações relacionadas ao desenvolvimento dos programas de diabetes e hipertensão, para análise das ações desenvolvidas, recursos disponíveis, resultados alcançados e definição de indicadores de avaliação dos programas, sob a forma de um sistema de informações, com acesso fácil e disponível. Foi desenvolvida uma proposta para análise dos programas baseada em um índice de efetividade e um indicador de custo da efetividade.

Para o estabelecimento de um indicador de efetividade, tornou-se necessário o estabelecimento de parâmetros de avaliação. A pesquisa de campo indicou a disponibilidade de alguns dados, que passaram a ser considerados para a elaboração de indicadores e posteriormente de uma fórmula agregadora.

A partir de um grupo de pessoas que faz parte de um programa de acompanhamento, dispomos de dados de óbitos, dias de internação e atendimentos.

Os números para o período de um ano do programa de diabetes e hipertensão indicam 354 óbitos, 10.244 dias de internação e 1.055.925 atendimentos (média dos anos 2000, 2001 e 2002).

Buscou-se uma fórmula que componha as três categorias, o que leva às seguintes considerações:

v óbitos, quanto MENOS, MELHOR;

v dias de internação, quanto MENOS, MELHOR;

$\checkmark$ atendimentos, quanto MAIS, MELHOR.

Interpretou-se a avaliação dos atendimentos "quanto mais, melhor", por representar investimentos em prevenção e tratamento ambulatorial, sugerin- 
do melhores condições de atendimento à população. Óbitos e internações já apresentam uma conotação negativa.

Tem-se que somar números relativos a essas três categorias, de acordo com alguma lógica, o que nos leva a fixar o seguinte:

v dado resultante, quanto maior (mais positivo), melhor a situação avaliada;

v a partir disso, os três indicadores devem obedecer à regra "quanto mais, melhor", logo, os dados de óbitos e internações devem ser invertidos em sua lógica (menos óbitos e dias de internação devem resultar em números crescentes positivos);

v deve haver proporcionalidade na contribuição de cada componente ao resultado final (um óbito deve pesar/influir mais fortemente que um dia de internação).

Para avaliar a proporção entre óbitos e dias de internação considerou-se que um óbito é um fato mais grave e, portanto, deve ter maior influência no cálculo do indicador de efetividade.

Para esse raciocínio, apelou-se para a NR-5, quadro 1-A (Segurança e Medicina do Trabalho, 1990), onde consta que uma morte equivale a 6 mil dias debitados, enquanto a perda de um dedo (não polegar) equivale a 300 dias debitados. Os dados servem para cálculo do índice de gravidade de acidentes em uma organização e devem ser entendidos como dias perdidos.

Deve-se salientar também que, para essa situação, estará identificada uma relação direta entre o fato causador e o efeito. Em nosso caso, das doenças crônico-degenerativas, dois aspectos diferenciais devem ser considerados:

v o óbito pode ter outras causas ou potencializadores, que não só a diabetes e/ou a hipertensão, ao contrário da relação morte-acidente;

v há uma relação entre o óbito e os dias de internação, já que os últimos muitas vezes precedem e prenunciam o primeiro, permitindo estabelecer uma equivalência, a partir da prática.

A partir do constatado no segundo item, cabe buscar a relação pretendida, isto é, responder à questão "quantos dias de internação equivalem a um óbito". Dito de outra forma, "o que deve ser considerado perda de mesma magnitude quando se quer medir a eficiência de um programa de controle de uma doença".

Dados de campo na cidade estudada apontam para uma média de 354 óbitos anuais e 10.244 dias de internação anuais, o que mostra uma relação de um óbito para 28,93 dias de internação. 
Para avaliar a proporção entre dias de internação e atendimentos, considerou-se que dias de internação são números negativos e os atendimentos são números positivos. Entende-se que um programa de prevenção a doença, quanto mais atendimentos fizer, melhores resultados (menos óbitos e dias de internação). Um dia de internação pode envolver vários profissionais e situações, vale dizer, enseja vários atendimentos.

Como ponto de referência, estabeleceu-se que um dia de internação é equivalente a 103,07 atendimentos. Esse valor resulta da divisão de 1.055.925 atendimentos por 10.244 dias de internação, o que replica o raciocínio usado quando da consideração de óbitos e dias de internação. Disso se deduz que um óbito deve equivaler a 2.981,81 atendimentos. Essas relações significam aceitar o que acontece na prática, no momento presente. Como montar a fórmula para cálculo/avaliação da efetividade do programa?

A fórmula deve ser construída de forma que seu resultado, quanto mais positivo, aponte para a efetividade (acerto) do programa. Assim somam-se dados de atendimento aos de óbitos e dias de internação. Os segundos devem ser invertidos em relação ao primeiro. Óbitos e dias de internação crescentes devem representar decréscimos no índice calculado, e atendimentos crescentes devem significar acréscimos.

A fórmula proposta pode, genericamente, apresentar-se da seguinte forma:

Índice de efetividade $=$ inverso de (óbitos + dias de internação) + atendimentos

Como as ordens de grandeza são diversas, cabe inicialmente equalizálas, a partir dos dados médios dos últimos três anos. Isso se justifica, dado que não caberia somar diretamente 354 óbitos com 1.055.925 atendimentos, pois os primeiros teriam quase nenhum efeito sobre o resultado.

\begin{tabular}{|ccccc|}
\hline 1) Óbitos & $\begin{array}{c}\text { 1b) Fator de } \\
\text { correção em relação } \\
\text { a atendimentos** }\end{array}$ & $\begin{array}{c}\text { 2) Dias de } \\
\text { internação }\end{array}$ & $\begin{array}{c}\text { 2b) Fator de } \\
\text { correção em relação } \\
\text { a atendimentos }\end{array}$ & 3) Atendimentos \\
\hline Multiplicar 1 por 3** & Multiplicar 2 por 3** & \multicolumn{2}{c|}{ Valor a corrigir 3** } \\
$1 / 354^{*}$ & 373.797 .450 & $1 / 10.244^{*}$ & 10.816 .895 .700 & 1.055 .925 \\
\hline
\end{tabular}

* A inversão do número foi feita para que seu efeito na fórmula se desse negativamente se o seu valor aumentasse e positivamente se decrescesse. O número de dias de óbitos e de dias de internações foi invertido para apresentar uma relação negativa na fórmula, quando associada ao número de atendimentos ambulatoriais.

** Esses fatores, multiplicados pelo valor a corrigir, conferem aos três elementos uma dimensão numérica equivalente. O valor a corrigir no qual o número de óbitos e número de internações foram multiplicados, foi o número de atendimentos ambulatoriais, por se tratar da maior grandeza. 
A partir desse ponto os três fatores componentes da equação têm dimensão equivalente. Deve-se ter em mente que os números iniciais apresentam dimensões com diferenças sem significado. Deve-se agora atribuir os pesos que representem as diferenças de valor que queremos atribuir, entre óbitos, dias de internação e atendimentos. Como anteriormente estabelecido:

、 1 óbito para 28,93 dias de internação;

- 1 dia de internação é equivalente a 103,07 atendimentos;

$\checkmark 1$ óbito equivale a 2.981,81 atendimentos;

v Índice de efetividade (Ie):

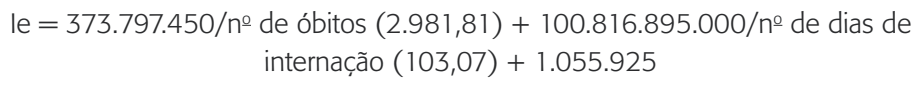

Dividir o resultado por 1.000 para ficar com grandezas ao redor de mil.

Outro dado levantado foi o valor dos recursos financeiros repassados para os programas. Foram definidos os valores repassados para os programas por meio da análise dos recursos repassados ao Fundo Municipal de Saúde. Levantou-se os repasses realizados pelo Ministério da Saúde, fundo a fundo, dos anos 2000 a 2002, e agrupados os valores correspondentes aos serviços nos quais os diabéticos e hipertensos estão inseridos por meio dos serviços utilizados e dos valores correspondentes aos medicamentos fornecidos. Devido à não-identificação dos valores correspondentes aos programas e serviços destinados ao hipertenso e ao diabético, optou-se por associar os repasses com os serviços que apresentam atividades destinadas ao programa. Também foram considerados os valores repassados pelo município de Itajaí e o percentual sobre a receita dos impostos recolhidos.

Em relação aos recursos financeiros investidos e ao índice de efetividade (Ie), espera-se que, quanto mais dinheiro investido, maior o índice Ie. Isso pode levar a várias situações. O dinheiro investido pode ser maior em determinado período e levar a um Ie maior (o que seria esperado) ou então a um Ie menor ou proporcionalmente menor.

Tal raciocínio é válido se a variável população permanecer constante. Se a população crescer, e se o investimento ou esforço no programa forem mantidos iguais, haverá uma tendência a um número maior de óbitos (por conta da maior população), o que pioraria o Ie. 
Levando em conta a variabilidade da população, devemos considerar este fator. Disso pode-se estabelecer uma relação reais investidos por habitante, que pode ser comparada ou relacionada com o Ie. Assim tem-se que:

Custo da efetividade $=\left(\mathrm{R} \$ / \mathrm{n}^{\circ}\right.$ - de habitantes $) / \mathrm{le}$

A análise do programa deve ser feita contemplando-se os dois indicadores: Ie, que será melhor quanto maior for; e custo da efetividade (representa o rendimento do investimento), quanto menor, melhor; isso significa dizer que cada ponto do Ie tem um custo, que quanto menor, melhor.

$\mathrm{O}$ indicador poderá ser acompanhado mensalmente. E anualmente a evolução e o desenvolvimento das variáveis que o contemplam. Podemos avaliar a aplicação do SIPDH em cada ano, conforme segue:

$\checkmark$ óbitos - quanto menor a quantidade de casos de óbitos, melhor o desempenho do município nas ações em saúde, resultados das ações;

、 média de dias de internação por diabetes e hipertensão - quanto menor o índice, melhor o desempenho em relação ao número de internações, complicações durante as internações, qualidade de vida do diabético e hipertenso, resultado do desempenho das ações ambulatoriais;

v atendimentos realizados em nível ambulatorial direcionados aos diabéticos e hipertensos - quanto maior esse índice, melhor o desempenho em relação às ofertas de serviços de prevenção, diagnóstico e tratamento ambulatorial;

- Ie - quanto maior, melhor o desempenho do município no ano analisado, levando-se em consideração a análise das variáveis comentadas anteriormente;

v recursos financeiros aplicados - quanto menor o índice, melhor a aplicação dos recursos financeiros em relação aos resultados alcançados, relação entre custo da efetividade.

\section{Aplicação do modelo de avaliação}

O modelo foi baseado nas equações propostas para avaliação por meio dos indicadores de morbidade, permanência hospitalar e alocação de recursos para os programas. Também foram consideradas as observações levantadas 
durante as entrevistas realizadas, para a análise dos problemas e necessidades apontados.

Foi realizado levantamento da série histórica de indicadores propostos entre 2000 e 2002. A escolha desse período se deu pela acessibilidade de informações coletadas na Secretaria de Saúde.

A análise dos dados apresentados na aplicação do modelo indicam que:

$\checkmark$ no ano 2000, o município de Itajaí apresentou o melhor índice de efetividade, em relação aos dois anos que seguiram. $O$ índice apresentado foi de 3,29 , e superou o padrão estabelecido de 3,16 ;

v no ano 2001, o índice apresentou queda em relação ao ano anterior, sendo apresentado o índice de efetividade 3,11.

- no ano 2002, o índice apresentado foi de 3,12, superior ao ano de 2001, porém inferior ao ano de 2000 e ao padrão estabelecido $(3,16)$.

Na variação entre os índices apresentados nos anos de 2000, 2001 e 2002 pode-se destacar:

v em 2000, o Ie foi o melhor dos anos analisados por ter apresentado variação pequena de óbitos em relação ao padrão (variação de dois óbitos a mais), menos dias de internação em relação ao grupo estudado (menos 1.194 em relação ao padrão), e uma redução de 2.545 atendimentos em relação ao padrão. Podemos considerar que apesar de um aumento de dois óbitos (+ 0,56\%), a redução dos dias de internação afetou consideravelmente para o bom desempenho do índice $(-11,65 \%)$. A redução de atendimentos não apresentou tanta relevância $(-0,24 \%)$. A sensibilidade combinada resultou em 4,13\%, o que significa um aumento em relação à situação anterior;

v em 2001, o Ie foi inferior aos anos 2000 e 2002 e padrão, Ie 3,11. Os óbitos apresentaram queda em relação ao padrão, aos anos 2000 e $2002(-16$, -18 e -30, respectivamente). As internações aumentaram em relação ao padrão em 876 casos, 2.070 em relação ao ano 2000, e 557 em relação ao ano 2002. Os atendimentos apresentaram redução na quantidade de 23.918 em relação ao padrão, 21.373 em relação ao ano 2000 e redução de 50.383 em relação ao ano 2002. Apesar da redução de casos de óbitos $(-4,51 \%)$, a elevação de dias de internação $(+8,55 \%)$ e redução de atendimentos $(-2,26 \%)$ foi considerável para a diminuição do índice em relação ao padrão e ao ano anterior. A sensibilidade combinada resultou em $-1,80 \%$, o que significa uma diminuição em relação à situação anterior; 
v em 2002 houve um aumento do Ie em relação ao ano anterior, porém abaixo do padrão e do ano 2000. Houve um aumento de óbitos em relação ao padrão em 14 casos, 12 em relação ao ano 2000 e 30 em relação ao ano 2001. Os dias de internação aumentaram 319 dias em relação ao padrão, 1.513 em relação ao ano 2000 e houve redução de 557 dias em relação ao ano 2001. Os atendimentos aumentaram em relação ao padrão, 26.465 atendimentos, 29.010 a mais do que o ano 2000 e 50.383 a mais do que o ano 2001. Pode-se observar que devido ao aumento dos óbitos (3,95\%), o índice não atingiu o padrão e apresentou-se superior ao ano anterior, os dias de internação também aumentaram em relação ao ano 2000 e padrão $(3,11 \%)$, porém reduziram em relação ao ano 2001. Os atendimentos aumentaram em relação aos anos anteriores e ao padrão (2,50\%). Assim, o Ie não alcançou o desejado, porém melhorou em relação ao ano anterior. A sensibilidade combinada resultou em menos $1,43 \%$, o que significa uma diminuição em relação à situação anterior.

Em relação ao custo da efetividade, podemos dizer que:

v no ano 2000 o custo da efetividade foi de $\mathrm{R} \$ 33,50$, no ano 2001 de $\mathrm{R} \$ 45,89$ e em 2002 de $\mathrm{R} \$ 53,52$;

v o ano 2000 apresentou o melhor le dos períodos analisados e o melhor custo da efetividade;

v no ano 2001 o custo da efetividade aumentou em relação ao ano anterior, houve redução do Ie e um aumento nos recursos direcionados aos programas, conseqüentemente, um pior resultado em relação ao ano anterior; isso significa que mais dinheiro gerou piores resultados;

v no ano 2002 o custo da efetividade aumentou em relação aos anos anteriores (pior resultado), houve um aumento do Ie em relação ao ano anterior, porém não alcançou o ano 2000 e o padrão, e os recursos investidos foram maiores do que nos anos anteriores.

\section{Conclusão}

Assim, analisando o número de óbitos, dias de internação e atendimentos, tem-se uma referência de desempenho do sistema de saúde e também do resultado do dinheiro investido. 
O acompanhamento anual da variação desse índice, possibilitará a análise do desempenho ao longo dos anos em relação aos programas específicos para hipertensos e diabéticos, da oferta de serviços destinadas ao grupo e da aplicação dos recursos financeiros. Pode-se avaliar a efetividade das ações e os recursos despendidos aos programas.

As fórmulas do indicador de efetividade e do custo da efetividade poderão ser aplicadas em diversos programas, considerando sempre um histórico para definir o peso de cada variável contemplada no serviço a ser avaliado.

Concluiu-se a pesquisa conforme os objetivos propostos, com um modelo para gestão em saúde pública, utilizando as fórmulas como indicadores para avaliação de qualidade e custo em saúde. Esse modelo apresenta-se como um Sistema de Informações para o Programa de Diabetes e Hipertensão (SIPDH).

\section{Referências bibliográficas}

BUSS, Paulo Marchiori et al. Sistemas de saúde: continuidades e mudanças - Argentina, Brasil, Chile, Espanha. São Paulo: Hucitec; Rio de Janeiro: Fiocruz, 1995.

CHIAVENATTO, Idalberto. Iniciação à administração geral. 2. ed. São Paulo: Makron Books, 1994.

CIANCIARULLO, Tamara. Indicadores de qualidade: uma abordagem perinatal. São Paulo: Ícone, 1998.

DEVER, G. E. Alan. A epidemiologia na administração dos serviços de saúde. São Paulo: Pioneira, 1998.

ERDMANN, Rolf Hermann. Organizações de sistemas de produção. Florianópolis: Insular, 1998.

LIMA, José Geraldo. Custos: cálculos, sistemas e análises. São Paulo: Atlas, 1979.

MARTINS, Domingos. Custos e orçamentos hospitalares. São Paulo: Atlas, 2000.

MINISTÉRIO DO TRABALHO. Segurança e medicina do trabalho. São Paulo: Atlas, 1990.

MORAES, Ilala Hammerli Sozzi de. Informações em saúde: da prática fragmentada ao exercício da cidadania. São Paulo, Rio de Janeiro: Hucitec, 1994.

SECRETARIA DO ESTADO DE SANTA CATARINA. Controle, avaliação e auditoria em saúde. Santa Catarina: SUS, 1994. 
- Informações básicas para gestores da Secretaria de Estado da Saúde de Santa Catarina. Secretaria do Estado de Santa Catarina, 2000.

TANAKA, Oswaldo Y. Avaliação de programas de saúde do adolescente: um modo de fazer. São Paulo: Universidade de São Paulo, 2001.

TINOCO, Aldo da Fonseca et al. Planejamento e administração de saúde. Brasília: Senado Federal, Centro Gráfico, 1984.

VALLA, Victor Vicent et al. Participação popular, educação e saúde: teoria e prática. Rio de Janeiro: Relume-Dumará, 1993. 